\title{
Overall design of radiation monitoring system formaritime nuclear facilities
}

\author{
Qingxin Lei ${ }^{1 *}$, Chenyu Shan ${ }^{1}$, Wenzhang Xie ${ }^{1}$ \\ ${ }^{1}$ China Nuclear Power Technology Research Institute, Shenzhen 518031, China
}

\begin{abstract}
The Radiation Monitoring System (RMS) is designed to check continuously the reactor at the operating set points, it assures that no individual and persons receive an unnecessary or hazardous exposure to radiation. According to the process and operation characteristics of Small Modular Reactor (SMR), these radiation monitoring channels can be grouped into the process radiation monitoring subsystem, the effluent radiation monitoring subsystem, the area radiation monitoring subsystem and the Post Accident Monitoring Subsystem (PAMS). Based on the architecture design of RMS, the demand of signal interface and power interface is analysed. Through introducing the marine environment, the problems of anti-shock, vibration, inclination and swing of loads are put forward.
\end{abstract}

\section{Introduction}

The maritime small modular reactor is based on mature and economic pressurized water reactor (PWR), which has the characteristics of high-power density, low fuel supply demand (long refuelling cycle), multi-purpose, clean and non-pollution. At present, offshore oilfields and island reefs are faced with power shortage and energy supply difficulties, which are difficult to meet the needs of potential development and engineering construction.

There is no mature technical scheme for maritime floating small modular reactor. Only Russia constructs the world's first Romonosov nuclear power icebreaker, which is based on two KLT-40S reactor units are designed with total power exceeding $70 \mathrm{MWe}$. The construction and commissioning is completed in 2018 , as supporting the strategic development and construction of Russia's Arctic Circle, the first maritime floating nuclear power plant goes to Pewick Port in Russia in the summer of 2019, providing guaranteed power for local residents, port facilities and petroleum resources exploitation activities.

The maritime small modular reactor has the characteristics of short construction period, low construction cost and low operation cost. Compared with the development of marine resources and the construction of island reefs base, it can not only meet the needs of stable power supply and continuous production of fresh water, but also improve its energy structure, contain diversification of energy supply and promote economic benefits.

\section{System Overview}

Radiation Monitoring System (RMS) can continuously monitor the overall radiative level of the reactor operation. During the radioactive leakage accident and post-accident, the abnormal change trend of radioactive level can be detected early, so as to ensure that the overall radioactive level of the reactor is maintained at the normal operation level, which protect the staff and the public from the risk of excessive radiation ${ }^{[1]}$.

According to the main monitoring function, RMS includes four subsystems. Some fixed radiation monitoring channels have continuous monitoring functions during accident and post-accident [2].

- The process radiation monitoring

- The effluent radiation monitoring

- The area radiation monitoring

- The post-accident radiation monitoring.

\subsection{Process Radiation Monitoring}

The process radiation monitoring subsystem has the functions of reactor safety barrier integrity monitoring and process system equipment radioactive leakage monitoring [3].

\subsubsection{Measurement from Fuel Element Cladding Damaged}

Fuel element cladding belongs to the first safety barrier of the reactor. After the cladding is damaged, radioactive activation products leak directly into the primary cooling system. Through by setting two monitoring channels, it can continuously monitor and indicate the $\gamma$ dose rate of characteristic nuclide fission products $(88 \mathrm{Kr}, 138 \mathrm{Cs}$, $137 \mathrm{Cs}$ ) in primary coolant. Once the fuel element cladding is damaged, then the radioactive data exceeds the preset alarm, and the isolation valve of the relevant system and the related pumps would automatically be interlocked off.

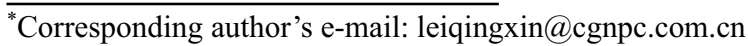


Simultaneously, it sends alarm signals to the main control room.

(1) Primary sampling system $\gamma$ dose rate monitoring channel;

(2) Chemistry and volume control system discharge coolant $\gamma$ dose rate monitoring channel;

\subsubsection{Steam Generator Leakage Rate Monitoring}

Under normal operating conditions of reactor, steam generator, the heat of reactor coolant core is transferred to secondary side medium by steam generator, then saturated steam is generated for power generation of turbo generator. As the primary coolant pressure boundary, it belongs to the second safety barrier of the reactor. When the heat transfer tube of evaporator is damaged, the primary coolant will directly enter into the secondary system and the main steam pipeline, and the contaminated saturated steam will be condensed from turbine gland system and released into the atmosphere, which may involve radioactive pollution to the environment. Through by setting monitoring channels to monitor $16 \mathrm{~N}$ and total $\gamma$ radioactivity in main steam and non-condensate gas radioactivity, the trend monitoring and indication of early leakage rate of steam generator are realized.

(1) Steam generator leakage rate and noble gas $\gamma$ radioactive monitoring channel;

(2) Non-condensate high humidity gas $\gamma$ radioactivity monitoring channel;

\subsubsection{Radioactivity Monitoring of Component Cooling Water System}

The component cooling water system, as a closed loop cooling water system, is an intermediate cooling water loop between the first loop and the final cold hydrazine. It can cool the heat exchanger and cooler of the first loop coolant system, secondary residual heat removal system, the chemistry and volume control system and other process systems. It also has the barrier isolation between the radioactive system and the external environment. The utility model can directly provide cold hydrazine protection from radioactive contamination after the steam generator heat transfer tube is damaged. Through by online continuous monitoring of component cooling water, early monitoring and judgment of equipment leakage point can be realized.

\subsubsection{Radioactivity Monitoring of Gaseous Radwaste System}

The gaseous radwaste system collects nitrogen, hydrogen and radioactive fission gas generated during the operation of the reactor process system, through on-line monitoring the radioactivity exhaust gas at the outlet of the delayed bed. When the radioactivity exceeds the preset alarm, the sound and light alarm is issued and the related isolation valve is automatically interlocked.

\subsubsection{Radioactive Activity Monitoring of Waste Water of Drainage Tank and Pit in the Assistant Engine Room}

Waste liquid drainage tank and pit collect the waste liquid. When the radioactivity of liquid radwaste exceeds the preset alarm, it reminds operators to start pump and put the radioactive waste liquid to the waste liquid treatment system.

\subsubsection{Chemistry and Volume Control System Filter Radiation Monitoring}

The filter is used to collect resin fragments and granular materials from the reactor coolant. When the fuel rod is damaged, the purifying circuit maintains $136 \mathrm{Cs}$ concentration of reactor coolant below the design requirements through redundant design of mixed resin bed and filter. Through by setting the "filter radiation monitoring channel" to remind service personnel that the filter capacity is about to meet the design requirements and needs to be replaced.

\subsection{Effluent Radiation Monitoring}

The effluent radiation monitoring subsystem provides radioactive information through continuous monitoring or sampling analysis, which is used to estimate and control the emission, concentration and total radioactive of the effluent to the environment, in order to protect the environment and public health.

\subsubsection{Radiation Monitoring of Gaseous Discharge}

Chimney is the only way for nuclear island cabins to emit radioactive gases into the atmosphere. The gas from reactor cabins, auxiliary cabins and other ventilation system is gathered to the chimney and then uniformly discharged environment. The radioactive activity of aerosol, radioactive iodine and noble gas in chimney are monitored continuously through by setting monitoring channel for the concentration of radioactive activity, which provided a basis for environmental assessment. Sampling devices are used as precise measurements and nuclide analysis for laboratory radiation monitoring.

(1) The monitoring channels of aerosol, radioactive iodine and noble gas radioactive activity concentration in chimney;

(2) Aerosol, radioactive Iodine, noble gas sampling device in chimney;

(3) ${ }^{3} \mathrm{H}$ and ${ }^{14} \mathrm{C}$ sampling devices in chimney;

\subsubsection{Radiation Monitoring of Liquid Discharge}

According to GB standards, drain tank is adopted for the waste liquid of nuclear island and secondary circuit. The waste liquid of the process system is collected in a certain volume storage pot, decayed and evenly mixed, then sampled to the laboratory for radioactivity measurement and nuclide analysis. Through by setting monitoring channels to continuously monitor the waste liquid, when 
it is found that unexpected high-level waste liquid enters the tank, the radiation instrument automatically sends out sound and light alarm, and interlock the isolation valve to prevent waste liquid from discharging.

(1) The monitoring channel of nuclear island liquid radwaste radioactive activity;

(2) The monitoring channel of secondary circuit liquid radwaste radioactive activity;

\subsection{Area Radiation Monitoring}

The area radiation monitoring subsystem can monitor and display the radiation data and air radiation level in the important area of the staff, so as to protect the power plant staff from unnecessary external and internal radiation. It is convenient for radiation protection person to quickly analyse, find and judge the possible radioactive leakage. It is generally composed of airborne radioactivity monitoring and $\gamma$ and $\mathrm{n}$ radiation monitoring ${ }^{[4]}$.

\subsubsection{Area Airborne Radioactivity Monitoring}

Through by setting continuous monitoring channels and sampling devices to monitor the radioactivity of air aerosols, radioactive iodine and noble gases in workplaces and equipment rooms, staffs are protected from radiation exposure and radioactive leakage of process system equipment is detected without delay.

(1) The monitoring channel of noble gas in ventilation system of control area;

(2) The monitoring channels for aerosols, radioactive iodine and noble gases in the reactor cabin;

(3) The monitoring channel of noble gas during refuelling discharge in the reactor cabin;

\subsubsection{Area $y$ and $n$ Radiation Monitoring}

Through by setting continuous monitoring channels to monitor the $\gamma$ dose rate in the workplace, the workers on the site are protected from radiation exposure. In addition, some channels can realize the radioactive monitoring of process equipment leaked during the normal operation and the post-accident, it is arranged in places where staff often go in and out, such as personnel corridors, control rooms and reactor halls.

(1) The monitoring channel of reactor cabin refuelling area $\gamma$ dose rate;

(2) The monitoring channel of spent fuel pool area $\gamma$ dose rate;

(3) The monitoring channel of reactor cabin $\gamma$ dose rate;

(4) The monitoring channel of main control room $\gamma$ dose rate;

(5) The monitoring channel of equipment and personnel passage in nuclear auxiliary cabin $\gamma$ dose rate;

(6) The monitoring channel of outside the reactor cabin n dose rate;

\subsection{During Accident and Post-Accident Radiation Monitoring (PAMS)}

The subsystem can not only monitor the level of radioactivity during the operation, refuelling and shutdown of the reactor, but also automatically implement interlock safety measures during the design basis condition and the design extend conditions, which can be used as a reference to evaluate and control the radiation leakage impact during accident and post-accident ${ }^{[5]}$.

(1) The monitoring channel of containment filtration exhaust system $\gamma$ dose rate during accident and postaccident;

(2) The monitoring channel of reactor cabin $\gamma$ dose rate during accident and post-accident (Shared);

(3) The monitoring channel of the chimney discharge radioactive during accident and post-accident (Shared);

(4) The monitoring channel of spent fuel pool area $\gamma$ dose rate during accident and post-accident (Shared);

(5) The monitoring channel of main steam radioactive during accident and post-accident (Shared);

(6) The monitoring channel of main control room radioactive during accident and post-accident (Shared);

\section{System Architecture}

The RMS system is divided into two levels (Level 0 and Level 1) according to the functional composition of the equipment (see Fig.1): 


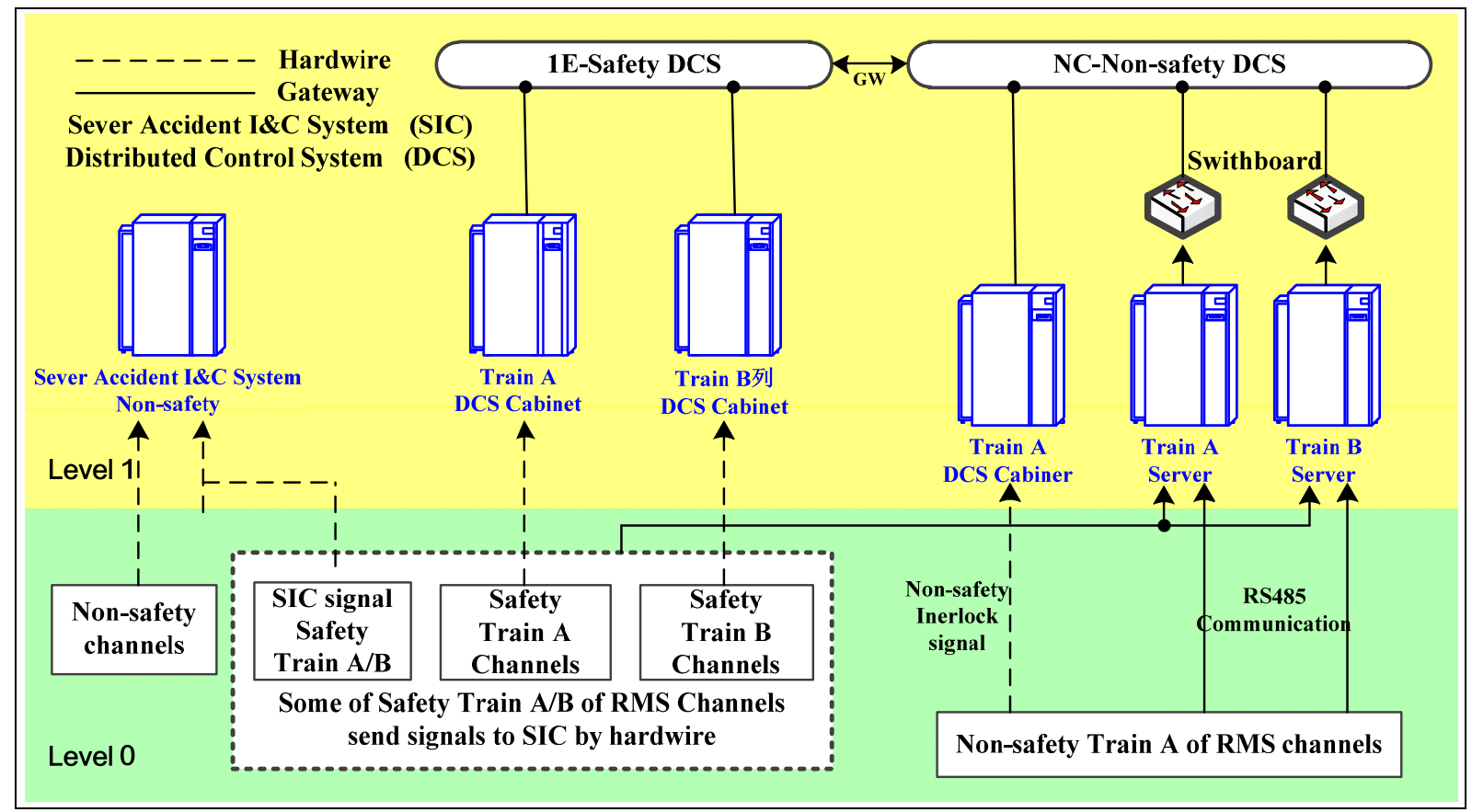

Fig.1 The system architecture of RMS

- Level 0: detector, local process or display unit, joint box and necessary sampling pump, control unit, etc.

- Level 1: data acquisition server, workstation;

Level 0 layer belongs to local processing or display equipment information, which is transmitted to cabinet and server respectively through by hard wiring and network protocol. Level 1 layer collects all radiation monitoring channels information for operators and radiation protection personnel to view, analyse and judge the reactor operation status. Safe related important radiation monitoring channel and automatic interlocking control signal are directly sent into cabinet by hard wiring and displayed in terminal of control room.

\subsection{Signal Interface}

The monitoring channel of RMS system can be divided into safety and non-safety. The analog and logic variable of the safe acquisition signal communicated with the hard wiring, then the analog and logic variable of the non-safe acquisition signal are communicated through the gateway. In addition, some non-safe logic signal alarm has the function of automatic interlocking protection, hard wiring is used to collect and communicate.

Some radiation monitoring channels need to be available under severe accident conditions. Hard wiring after signal isolation is sent directly to severe accident instrument \& control system for acquisition, monitoring and display.

\subsection{Power Interface}

The power level of RMS system is 220VAC, the power interface diagram is shown in Fig.2.
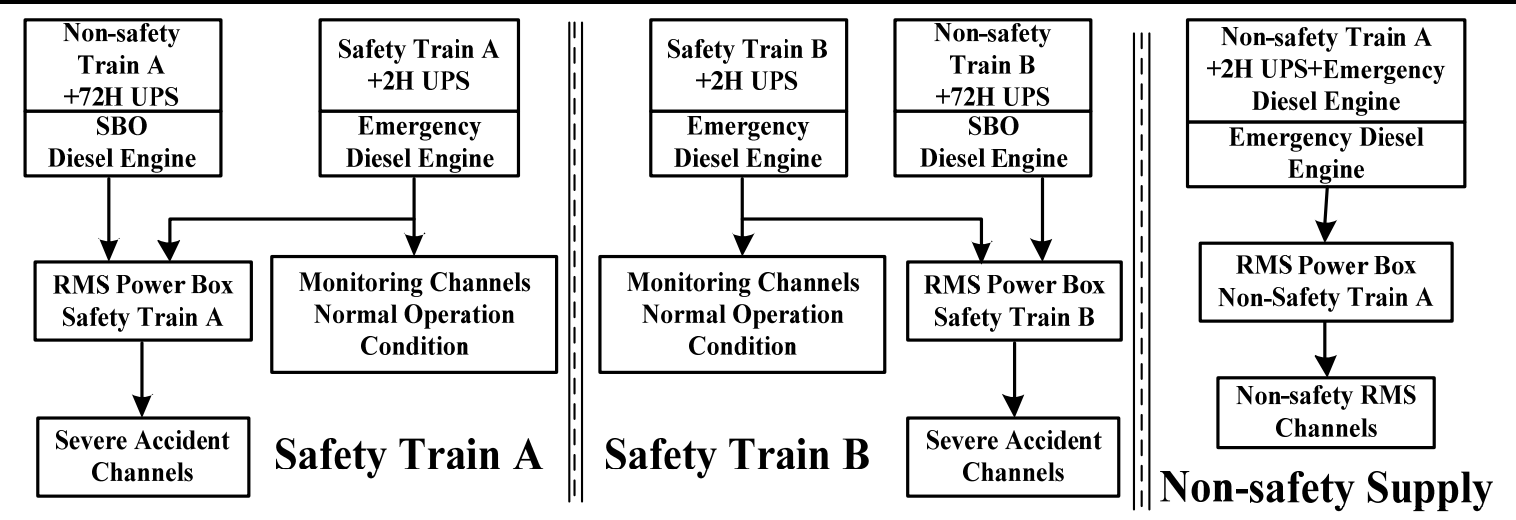

Fig.2 The power interface of RMS

The non-safety radiation monitoring channel is powered by 2-hour UPS and emergency diesel engine of the non-safety train A.

The operating conditions of safety radiation monitoring channel can be divided into three types: design basis accident, severe accident and Ship Black Out (SBO).

(1) Under the design basis accident condition the safety radiation monitoring channels are powered by 2 hours UPS and emergency diesel engine.

(2) Under severe accident conditions, the related 
radiation monitoring channels are powered by 72 hours UPS of non-safety.

(3) Under SBO condition the radiation monitoring channels are powered by emergency diesel engine generator.

\section{System Characteristic}

Based on the mature PWR technology for maritime small module reactors, the function of radiation monitoring system is basically similar to that of nuclear power plants on land. Considering that the slope, swing, and anti-shock of the sea are different from those of the nuclear power plant on land, the radiation monitoring equipment follow to the different rules and regulations.

\subsection{Regulation Standard}

\begin{tabular}{ll}
\hline \multicolumn{1}{c}{ Standard Name } & Standard Number \\
\hline $\begin{array}{l}\text { Design and Construction Rules for } \\
\text { Electrical Equipment on Nuclear Island }\end{array}$ & RCC-E/ 2005 \\
of PWR Nuclear Power Station \\
(France)
\end{tabular}

\subsection{Equipment Supervision}

Non-safety equipment in RMS system is supervised by China Register of Shipping (CCS), and safety equipment is supervised by National Nuclear Safety Administration.

The non-safety radiation monitoring equipment should be taken certificate of approval according to CCS supervision catalogue.

The safety radiation monitoring equipment only meets CCS specifications when it is put into operation on the sea, without seismic requirements. In addition, some safety equipment needs to be available during refuelling at the dock. They need to meet the environmental requirements of anti-earthquake, ocean anti-shock, slope and swing.

\subsubsection{Anti-Shock Load}

Load input values for anti-shock test of marine reactor cabin outside are determined as follows:

Table 1 Anti-shock parameters

\begin{tabular}{lll}
\hline Spectral Type & Duration & Peak Acceleration \\
\hline Type L spectrum & $22 \mathrm{~ms}$ & $7 \mathrm{~g}$ \\
Type I spectrum & $11 \mathrm{~ms}$ & $10 \mathrm{~g}$ \\
Type II spectrum & $6 \mathrm{~ms}$ & $30 \mathrm{~g}$ \\
Type III spectrum & $2.3 \mathrm{~ms}$ & $50 \mathrm{~g}$ \\
Note: $1 . \quad$ L: One typical spectrum for shocks with long \\
duration and low peak acceleration. \\
2. I: One typical spectrum for shocks with long \\
duration and relatively low peak acceleration. \\
3. II: One typical spectrum for shocks with medium \\
duration and medium peak acceleration. \\
4. III: One typical spectrum for shocks with short \\
duration and high peak acceleration.
\end{tabular}

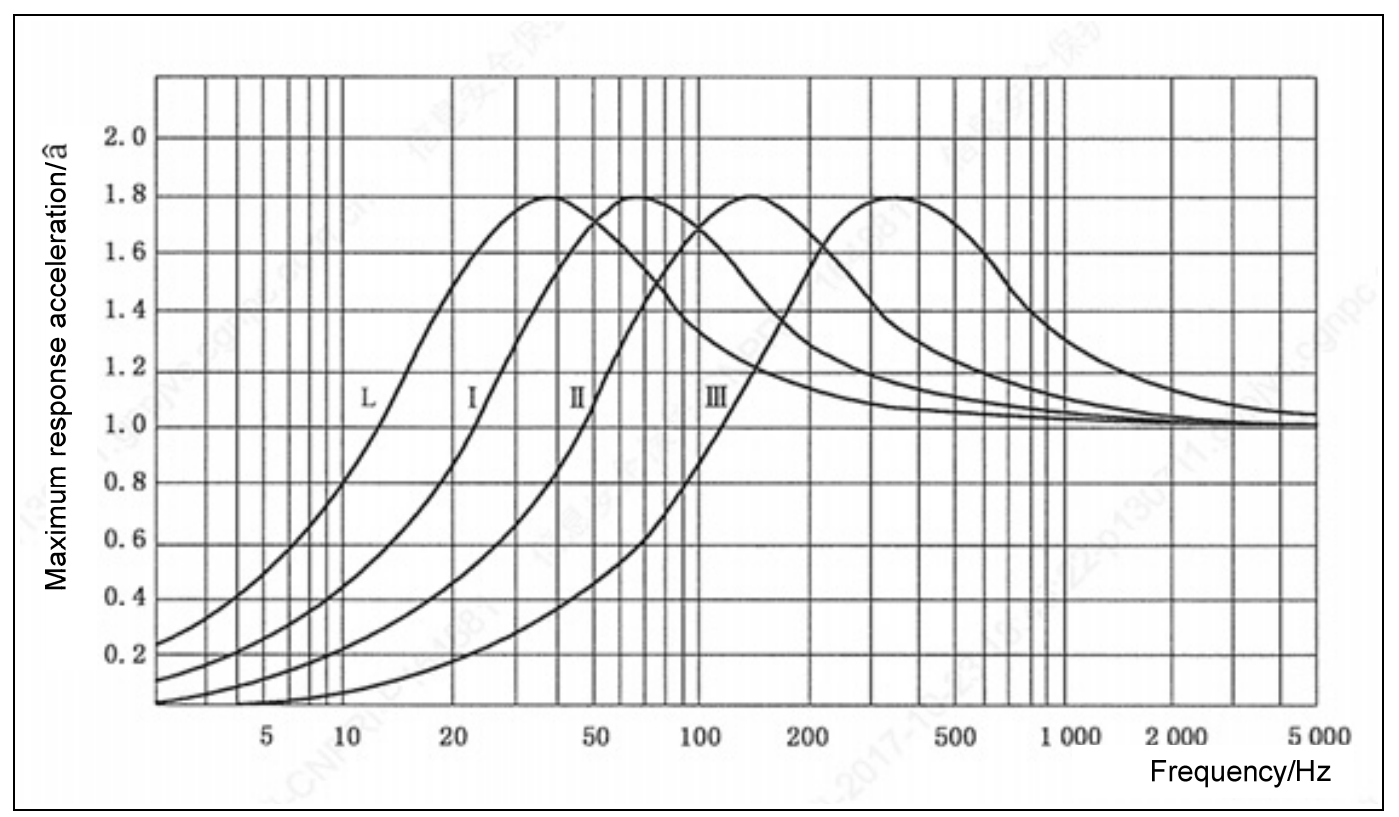

Fig.3 Typical shock response spectrum

Among the selection of four spectral types, it is suggested to select the spectral type with the maximum absolute acceleration response corresponding to the first natural frequency of the item.

\subsubsection{Constant Acceleration Load}

The equipment should be kept available under constant 
acceleration. The parameters are shown in Table 2 .

Table 2 Constant acceleration parameters- constant acceleration

\begin{tabular}{lll}
\hline Physical Parameters & Unit & Grade(M2) \\
& & \\
\hline X axial acceleration & $\mathrm{m} / \mathrm{s}^{2}$ & 5 \\
Y axial acceleration & $\mathrm{m} / \mathrm{s}^{2}$ & 6 \\
Z axial acceleration & $\mathrm{m} / \mathrm{s}^{2}$ & 5 \\
\hline
\end{tabular}

\subsubsection{Slope and Swing Load}

The equipment should be kept available under constant acceleration. The parameters are shown in Table 3.

Table 3 Slope and swing parameters

\begin{tabular}{llll}
\hline $\begin{array}{l}\text { Environment } \\
\text { Parameters }\end{array}$ & Physical parameters & angle & cycle \\
\hline tilt & Heel angle & $22.5^{\circ}$ & - \\
& Inclination angle & $22.5^{\circ}$ & - \\
swing & Heel angle & $22.5^{\circ}$ & $5 \mathrm{~s}$ \\
Note:1. Horizontal and vertical inclination & may & occur \\
simultaneously; & Inclination angle & $22.5^{\circ}$ & $5 \mathrm{~s}$ \\
2. "“-” means no; & & \\
3. All safety level radiation monitoring equipment \\
should be checked and calculated by $45^{\circ}$ rolling. \\
\hline
\end{tabular}

\section{CONCLUSION}

Maritime nuclear facilities use the PWR technology, the operation condition and severe accident condition have been considered in the system design scheme, the signal interface and power interface can meet the functional requirements. Process radiation monitoring, effluent radiation monitoring and area radiation monitoring are carried out respectively according to regulation standard. Furthermore, considering the differences between marine environment and land, the availability of equipment under different load requirements is proposed for equipment identification test.

Small offshore reactor is a nuclear power plant built on offshore platforms or hulls. It can provide reliable power and desalination for islands and offshore oil drilling platforms. In addition, its good mobility characteristics could eliminate the rigorous site selection problems and population relocation faced by current onshore nuclear power plants, there will be great prospects in some coastal city.

\section{References}

1. Guangdong Nuclear Power Training Center, 900MW PWR nuclear power plant system and equipment[M], China Beijing: Atomic Energy Press, 2005:296-304.

2. Sun Hanhong, Cheng Pingdong etc. Third generation nuclear power technology AP1000[M], China Beijing: China Electric Power Press, 2016:394-397.

3. Lingqiu, Guo Lanying, Li Dongyu, etc. Radiometric measurement technology for nuclear power plants $[\mathrm{M}]$,
China Beijing: Atomic Energy Press, 1998:162-167.

4. John R. Lamarsh, Anthony J. Baratta, Introduction to Nuclear Engineering[M], America New Jersey:Prentice-Hall, Inc., 2001: 535-539.

5. Mitica Dragusin, Doru Stanga, et al. Radiation monitoring under emergency conditions [J]. Romanian Journal of Physics, 2014,59(Nos. 910):891-903 . 